\title{
Aus Fehlern lernen
}

\section{Aufgaben des Qualitätsmanagements in Jugendämtern}

\author{
Timo Ackermann, Felix Brandhorst, Reinhart Wolff
}

Prof. Dr. Reinhart Wolff ist

Hochschullehrer, Timo Ackermann und Felix Brandhorst sind wissenschaftliche Mitarbeiter an der Alice Salomon Hochschule Berlin. Internet http://www.ash-berlin.eu
Die Jugendämter sind die zentralen Instanzen im Kinderschutz. Sie sollten deshalb über ein Qualitätsmanagement verfügen, das falsche Entwicklungen entdecken und korrigieren kann. Denn der »Fehler" einer Fachkraft steht erst am Ende einer Fehlerkette, er wird überhaupt erst möglich in einem Fehler begünstigenden Milieu.

Kinderschutzfehler sind in aller Munde und die Debatte über sie entfacht sich erneut an jedem neuen massenmedial verhandelten Fall von Gewalt gegen Kinder. Ob über Fälle von Kindesmisshandlungen mit Todesfolge berichtet wird, für die Namen wie Kevin (Bremen, 2006) und Lea-Sophie (Schwerin, 2007) stehen, oder über Fälle sexuellen Missbrauchs, wie in jüngster Zeit in Bezug auf katholische Bildungseinrichtungen und Geistliche geschehen - hinter all diesen Debatten stehen dieselben Fragen: Wieso hat niemand die Gewalthandlungen verhindert? Wer trägt die Verantwortung für diese Fehlleistungen? Und wie kann aus den Fehlern gelernt werden, um in Zukunft den Schutz von Kindern vor Gewalt besser zu gewährleisten? Diese Fragen richten sich auch und gerade in Fällen, in denen zu Schaden gekommen Kinder unter staatlicher Fürsorge standen, an die Jugendämter als zentrale Instanzen im Kinderschutz.

Damit Jugendämter und andere Kinderschutzorganisationen aus ihren Fehlern lernen können, muss aber zunächst geklärt werden, was als Norm definiert und welche Abweichung als Fehler gewertet wird. Dieser Klärungsprozess bezieht sich auf zwei Ebenen:

- Erstens muss verhandelt werden, welches Verhalten anhand welcher Kriterien als Kindeswohlgefährdung und Kindesmisshandlung beurteilt werden kann.

- Zweitens muss ermittelt werden, wie Kinderschutz-Organisationen angemessen auf Kindeswohlgefährdungen reagieren können und an welchen fachlichen Standards sich die Praxis bemessen lässt.

Dies bedeutet im sozialarbeiterischen Kontext vor allem, dass geklärt werden muss, auf welchem Wege Fachkräfte im Einzellfall zu einer Problemkonstruktion finden können, die Handlungsspielräume zur Konfliktlösung eröffnet und in der die (Beteiligungs-) Rechte der Betroffenen berücksichtigt werden.

Insofern verweist die Frage nach Fehlern im Kinderschutz auf zwei zentrale Problembereiche: auf die Diagnostik im Kinderschutz und die methodische Angemessenheit und Wirksamkeit des Hilfeprozesses zum Schutz von Kindern vor Gewalt und vor Vernachlässigung.

Denn so eindeutig in der öffentlichen Wahrnehmung die "Diagnose « auch ausfällt, was eine Misshandlung und (begrifflich noch unpräziser) was eine Kindeswohlgefährdung ist, so schnell wird dieses klare Bild bei genauerer Betrachtung erschüttert. Beispielsweise geriet der schließlich auch des sexuellen Missbrauchs beschuldigte Augsburger Bischof Mixa zunächst in die Schlagzeilen, weil er Kinder geschlagen haben sollte und er einräumte, »die ein oder andere Watsch'n « (1) verteilt zu haben, sehen wir doch schnell, dass historisch relative Werturteile in der Beurteilung solcher Vorwürfe im Rückblick eine Rolle spielen. Denn eine "Watsch'n « könnte man als gewaltsame Handlung bezeichnen, die wahrscheinlich vom Großteil der heutigen Leserinnen und Leser eines Feuilletons als von der Norm abweichendes Erziehungsmittel wahrgenommen wird. Die erteilte Ohrfeige liegt in diesem Fall jedoch etwa 30 Jahre zurück. Ruft man sich in Erinnerung, dass das Recht auf eine gewaltfreie Erziehung erst im November 2000 gesetzlich verankert wurde, dann lässt sich attestieren, dass eine »Watsch'n « zum Zwecke der Erziehung von Kindern in den 1970er Jahren nicht nur juristisch, sondern auch gesellschaft- 
lich noch weithin akzeptiert war und nicht durchgängig als Kindeswohlgefährdung oder Kindesmisshandlung gewertet wurde. (2)

Die hier erörterte historische Perspektive verdeutlicht, dass das Feststellen einer Kindesmisshandlung immer eine auf Wahrnehmung gegründete Deutung beinhaltet, somit eine gedankliche Konstruktion von Menschen ist, auf die unentwegt Veränderungen von rechtlichen, wissenschaftlich-fachlichen und moralischen Normen einwirken.

Kindeswohlgefährdungen und Kindesmisshandlungen sind also keine feststehenden Tatsachen, sondern kontextgebundene Phänomene, die von soziokulturellen Bedingungen, konkreten Situationen und in der Bewertung zudem von unterschiedlichen fachlichen Blickwinkeln (juristischer, kriminologischer, medizinischer, psychologischer, sozialwissenschaftlich-sozialpädagogischer Blickwinkel) abhängig sind. All diese verschiedenen Perspektiven und Bedingungen müssen berücksichtigt werden, um im Einzellfall zu einer Diagnose zu gelangen. Und erst auf Grundlage dieser mehrseitigen Konstruktionsleistung ist es dann auch möglich, Fehler im Kinderschutz $\mathrm{zu}$ identifizieren, sie $\mathrm{zu}$ untersuchen und aus ihnen zu lernen, um Kinder effektiver vor Gewalt zu schützen.

\section{Wie und wozu aus Fehlern lernen im Kinderschutz?}

Die Frage, wie im Kinderschutz aus Fehlern zu lernen sei, gewann angesichts von vermehrter Aufmerksamkeit (vgl. Ackermann 2010) und medial skandalisierten Kinderschutzfällen an politischer und professioneller Bedeutung (vgl. Biesel 2008, Wolff 1997). Nicht zuletzt deswegen hat die Frage, die wir in dem Bundesmodellprojekt "Aus Fehlern lernen. Qualitätsmanagement im Kinderschutz« verfolgen (vgl. B 2009; Biesel 2010), an Brisanz gewonnen.

Alltäglich erfahren wir, dass ganz grundsätzlich die Möglichkeit besteht, aus eigenen Fehlern zu lernen: Wir erkennen, dass wir gute Ergebnisse nicht erzielten, die wir angestrebt haben, dass wir etwas übersehen oder falsch gemacht haben, dass wir eine eigene oder fremde Erwartung "frustriert " haben (vgl. aus lerntheoretischer Perspektive Oser/Hascher/Spychiger 1999).
Aber auch aus den Fehlern anderer können wir »advokatorisch « (ebd.) lernen: Wir erkennen, dass das Handeln A, einer anderen Person, in der Situation B nicht die gewünschte Folge $C$ hervorgebracht hat.

Diese Formulierung deutet schon an, wie schwierig es ist, im Kinderschutz aus Fehlern zu lernen: Denn die Situationen und Handlung sind nicht einfach miteinander $\mathrm{zu}$ vergleichen und einfach Ursache-Wirkungs-Schemata können kaum Gültigkeit beanspruchen. Jede Fallsituation hat ihre eigene (systemische) Logik.

Wie kann eine Fachkraft im Kinderschutz entscheiden, ob die aktuelle Situation eine Situation des Typs B ist, oder eine des Typs A? Und im Übrigen: Welches Handeln zur Verbesserung der Situation geführt hat, lässt sich oft erst im Nachhinein entscheiden; und auch dann können immer noch unterschiedliche Beobachter unterschiedlicher Ansicht sein (vgl. Ackermann 2010).

Dieses Risiko müssen Fachkräfte täglich, in einer Güterabwägung (z. B. Schutz des Kindes vor weiterer Misshandlung vs. Schutz der Beziehungen zu Eltern, Freunden und Geschwisterkindern) und vor allen Dingen fachlich gekonnt balancieren. $\mathrm{Zu}$ ihrem eigenen Schutz, aber auch zu dem der Hilfeteilnehmerinnen und Hilfeteilnehmer (Nutzerinnen und Nutzer sozialer Dienste), müssen sie diese Risikoabwägung nun auch ausführlich dokumentieren, was einerseits hilft, Entscheidungen mit schwerwiegenden Konsequenzen mit größerer Sorgfalt zu treffen, andererseits gerade unter Rechtfertigungszwang - einen Nachweis fachlicher Kompetenz und Sorgfalt führen zu können.

Wozu sollte aber aus Fehlern gelernt werden? Zum Ersten ermöglicht das Betrachten von Fehlern den Rückschluss auf Qualität. Wenn wir eine Normabweichung - einen Fehler - bestimmen, müssen wir auch die Norm - die Qualität bestimmen. Darin liegt eine große Chance: Denn oft ist im Kinderschutz, gerade in der Kooperation verschiedener Professionen nicht klar, was eigentlich die Qualität in der Kinderschutzpraxis sein kann. Sich über die Norm (guter oder sogar bester Fachpraxis) im Kinderschutz zu verständigen, ist darum ein erster Schritt, um aus Fehlern zu lernen und ein erstes Ergebnis (Produkt), das sich aus der Arbeit an Fehlern ergeben kann: Wenn wir in den Dialog über Fehler und Qualität im Kinderschutz treten, lernen wir, was wir im Kinderschutz wie tun können und müssen.

Zweitens ermöglich das Lernen aus Fehlern den Aufbau negativen Wissen: Wir erkennen, dass ein bestimmtes Verhalten zu einer negativen Folge führt (vgl. nur Oser/Hascher/Spychiger 1999). Ein bekanntes Beispiel: Ein Kind beobachtet, dass eine Person die Straße bei rotem Ampellicht überquert und beinahe von einem Auto erfasst wird. Das Kind kann lernen, im Sinne eines negativen Wissens, dass die Straße bei rotem Licht zu überqueren Gefahr bedeutet. Es lässt sich allgemein gesprochen lernen, dass ein Verhalten zu einem unerwünschten Ergebnis führt.

So weit die Theorie - für die Praxis müssen wir einschränkend sagen, dass wir im Kinderschutz - trotz der wachsenden Begeisterung für sogenannte Ampelmodelle - nicht einfach erkennen können, »ob die Ampel auf rot oder grün steht «. Vertraue ich der Familie, dass sie die Vereinbarungen einhält, oder muss ich kurzfristig intervenieren? Fehler und Erfolg liegen bei solchen Abwägungen nah beieinander.

Bislang haben wir nur über professionelle Fehler gesprochen, Fehler, die auf der Ebene der Entscheidung liegen. Nehmen wir jedoch aktuelle Fehlermodelle zur Hand sehen wir, dass Fehler immer auf mehreren Ebenen zu suchen sind (vgl. Reason 2008). Vielmehr noch: Der Fehler einer Fachkraft steht erst am Ende einer Fehlerkette, er wird überhaupt erst möglich in einem Fehler begünstigenden Milieu.

In einem systemischen Fehlermodell müssen wir in den Blick nehmen, dass Fehler auf den Ebenen der Zusammenarbeit zwischen den Organisationen, auf der Ebene der Kinderschutzorganisation selbst und auf der Ebene der Teams in der Kinderschutzorganisation eine mindestens ebenso bedeutende Rolle spielen wie die Entscheidungen der einzelnen Fachkräfte.

Nicht zuletzt prägen gesellschaftliche Rahmenbedingungen und sozialpolitische Entscheidungen die Kinderschutzpraxis entscheidend. (3) Wenn wir aus Fehlern lernen wollen, beispielsweise auch in der Analyse des einzelnen Falls, müssen diese Ebenen berücksichtigt werden. Kommen sie nicht vor, wird man der Komplexität der Erzeugung von Fehlern im Kinderschutz nicht gerecht. 


\section{Das Lernen aus Fehlern als Aufgabe des Qualitäts- managements im Jugendamt}

Qualitätsmanagement im Jugendamt greift natürlich die Frage des Lernens aus Fehlern auf, ist aber nach unserem Verständnis breiter anzulegen. Qualitätsmanagement sehen wir, auch im Rahmen des Bundesmodellprojektes, als ein Konzept und Verfahren, das sich mit der gezielten Bewertung und Weiterentwicklung von sozialen Dienstleistungen befasst (vgl. Merchel 2010: 15).

Das Ziel ist nicht die Erarbeitung neuer Checklisten und Verfahrensvorschriften, die in Schubladen verschwinden oder sogar zu »Dokumentenfriedhöfen « werden. Vielmehr geht es darum, Entwicklungen von Organisationen anzustoßen. An zwölf Modellorten erproben wir daher, aufbauend auf den Erfahrungen des Kronberger Kreises für Qualitätsentwicklung e. V., dialogischen Settings der organisationalen Selbstveränderung. Wichtig um womöglich gar eine Null-Fehler-Kultur zu erreichen. Vielmehr kann das Identifizieren, Bewerten und Bearbeiten von Risiken und Fehlern (vgl. die Broschüre des Landes NRW: MGFFI-NRW 1999) Anlass zur Entwicklung der Kinderschutzorganisation geben. Grundlage dafür ist eine fehlerfreundliche Organisationskultur. Die leitende Frage in einer solchen Kultur ist: »Wie entstand der Fehler? « und nicht: "Wer hat den Fehler gemacht? « Die erste Frage zielt auf die Analyse von Ursachen, die zweite Frage auf die Zuschreibung von Schuld.

Gelingt es, eine Organisationskultur zu schaffen, in denen Fachkräfte über ihre Praxis, ihre Kommunikationsformen und Beziehungen sprechen, dann ist eine methodische, instrumentelle und organisatorische Unterfütterung dieser Kultur sinnvoll. Umgekehrt kann aber auch die Einführung solcher Instrumente eine fehlerfreundliche Kultur begünstigen, sie kommt aber nicht ohne sie aus. Wir nennen einige Ansätze, die sich in der Luft-

\section{»Im Qualitätsmanagement geht es um die Analyse von Ursachen, nicht um die Zuschreibung von Schuld «}

Dienstleistungen lassen sich Lernhinweise, nicht zuletzt für Kinderschutzorganisationen, gewinnen, vgl. Merchel 2005: 186 ff. und: Amt für Soziale Dienste Bremen/Kronberger Kreis $f$. QE 2010)

- Rituale in Teams und Arbeitsgruppen (z. B. stellen die Fachkräfte regelmäßig ein Buch vor, das an einen eigenen Fehler (oder Erfolg) erinnert, oder Fehler und Beinahe-Fehler stehen als Standardtagesordnungspunkt auf der Tagesordnung (vgl. ebd.: 189 f.; vgl. a. Bellabarba 2008)

- Schaffung einer Stelle oder Arbeitsgruppe für Qualitätsentwicklung und Qualitätsmanagement, für das Lernen aus Fehlern und Erfolgen, die Fehler und Erfolge laufend beobachtet, einen jährlichen Fehlerbericht verfasst, die Ergebnisse in der Organisation verbreitet und auf der Organisationsebene Vorschläge zur Qualitätsentwicklung unterbreitet

- Risikobewertungen und Fehleranalysen (multiprofessionelle Risikobewertungen bezogen auf die Prozesse der Zusammenarbeit, durchgeführt in regelmäßigen Abständen unter Einbeziehung professioneller Kooperationspartner, aber auch Analysen innerhalb von Organisationen und Teams, unter Einbeziehung aller Hierarchieebenen, anhand von kritischen Ereignissen in Fällen, aber auch anhand von Fällen, die im »Normalbetrieb « verliefen)

\section{Fazit und Ausblick: Das Lernen aus Fehlern als Chance für die Qualität im Kinderschutz} machen, um von Kooperationspartnern, Hilfeteilnehmenden und den eigenen Mitarbeitenden zu lernen. Die Chance besteht in einem solchen Setting nicht zuletzt darin, etwas über die eigenen Fehler und Erfolge zu lernen, um so die Qualität in der Arbeit weiter entwickeln und Fehler vermeiden zu können.

Dass das Hauptinteresse eines Qualitätsmanagements auf der gemeinsamen Bewertung und Entwicklung von Qualität liegen sollte (vgl. Merchel 2010), gilt unserer Ansicht nach auch mit Blick auf das im Kontext des Kinderschutzes vielfach diskutierte Thema des Risiko- und Fehlermanagements. Es kommt weniger darauf an, jeden Arbeitsschritt oder jede Fachkraft auf Fehler hin zu kontrollieren, fahrtindustrie, aber besonders in der Medizin und in der Pflege durchgesetzt haben. Die Ansätze können von den Fachkräften genutzt werden und im Rahmen eines Qualitätsmanagements einund durchgeführt werden:

- Fehlerberichtssysteme, Erstellung von Fehlerlisten (organisationsbezogene, aber auch teaminterne, z. B. nur ein Zettel oder Mikroartikel mit »Fehler « überschrieben, wo Teammitglieder Unfälle oder kritische Ereignisse vermerken, die fast zu schlimmeren Fehlern geführt hätten (vgl. Bellabarba 2008), bis hin zu elektronischen, überregionalen und anonymen Systemen von Fehlerberichtssystemen, vgl. Internet http://www.kritische-ereignisse.de)

- Einführung eines Beschwerdemanagements (z. B. aus den Beschwerden der Nutzerinnen und Nutzer sozialer
Mit diesen ersten Hinweisen können wir nur andeuten, welche Möglichkeiten es gibt, das Lernen aus Fehlern im Sinne eines Qualitätsmanagements $\mathrm{zu}$ befördern. Dabei ist deutlich geworden: Es gibt doch einige Ansätze, die bereits jetzt und überall umgesetzt werden können.

In dem von uns durchgeführten Modellprojekt »Aus Fehlern lernen. Qualitätsmanagement im Kinderschutz« erproben wir derzeit gemeinsam mit den Fachkräften verschiedener Professionen, aber auch gemeinsam mit Hilfeteilnehmern und Nutzern Modelle der dialogischen Auseinandersetzung über Fehler. Schon jetzt zeichnet sich ab, dass wir nach dem Projekt nicht stehenbleiben 
können. Die vielversprechenden Ansätze, die wir vielerorts sehen, müssen weiter geführt werden, durch die Fachkräfte selbst, aber auch mit einer Begleitung von außen. Denn es zeigt sich auch: Das Sprechen über Fehler fällt schwer. Eine fehleroffene Kultur zu erreichen, braucht Zeit - gerade im Kinderschutz. Sie lässt sich nicht von oben verordnen. Im gemeinsamen Lernprozess, im interprofessionellen Dialog gelingt das besser, wie sich in unserem Projekt zeigte. Denn wir konnten bürokratische Engführungen im
Fehler- und Risikomanagement vermeiden und auf dem Weg zu einer achtsamen Kinderschutzkultur bereits jetzt Entwicklungen anregen, die nach dem Ende des Modellprojektes weiter dazu beitragen werden, die Qualität in Kinderschutzprozessen zu verbessern: Entwicklung einer ganzheitlichen demokratischen Kinderschutzkonzeption zur Sicherung des Kindeswohls, des Eltern- und Familienwohls und des Gemeinwohls, gemeinsame Qualitätsentwicklung von Fachkräften und Hilfeteilnehmern und Hilfeteil-

\section{Literatur}

Ackermann, Timo (2010): Krise, Risiko und Selbstschutz im Kinderschutz. In: Forum Kinder- und Jugendarbeit 2/2010. Im Erscheinen.

Amt für Soziale Dienste Bremen/Kronberger Kreis für Qualitätsentwicklung e. V. (2010). Qualitätssicherung und Risikomanagement im Kinderschutz. Das Bremer Konzept. Bremen: AfSD.

Bast, Heinrich, Bernecker Angela, Kastien, Ingrid, Schmitt, Gerd u. Wolff, Reinhart (1975): Gewalt gegen Kinder. Kindesmißhandlung und ihre Ursachen. Reinbek bei Hamburg: Rowohlt.

Bellabarba, Julia (2008): Qualitätsmanagement Toolbox (2). In: Forum für Kinderund Jugendpsychiatrie, Psychosomatik und Psychotherapie 2 - 2008. Internet http:// www.bkjpp.de/forum/forum_2008_2.pdf, 18. Mai.: S. 80-83.

Biesel, Kay (2010): Aus Fehlern lernen. Qualitätsmanagement im Kinderschutz. Erste Einblicke und weiterführende Ausblicke. In: Das Jugendamt. Nr. 3/2010. S. 111114.

Ders. (2008): Zwischen Fehlervermeidung und -Offenheit. Wo stehen die sozialen Hilfesysteme? In: Sozial Extra 11 und 12/2008.

Castel, Robert (2005): Die Stärkung des Sozialen. Leben im neuen Wohlfahrtsstaat. Hamburg.

MGFFI-NRW (Ministerium für Generationen, Familie, Frauen und Integration des Landes Nordrhein-Westfalen) (2009): Risikomanagement bei Kindeswohlgefährdung. Kompetentes Handeln sichern. Düsseldorf.

Merchel, Joachim (2010): Qualitätsmanagement in der Sozialen Arbeit. Eine Einführung. Weinheim und München, 3. überarbeite. Auflage.

Ders. (2005): Organisationsgestaltung in der Sozialen Arbeit: Grundlagen und Konzepte zur Reflexion, Gestaltung und Veränderung von Organisationen. Weinheim und München.

Swift, Karen J., Callahan, Marilyn (2009): At Risk. Social Justice in Child Welfare and Other Human Services. Toronto, Buffalo, London: Toronto University Press.

Oser, Fritz; Hascher, Tina; Spychiger, Maria (1999): Lernen aus Fehlern. Zur Psychologie des »negativen « Wissens. In: Althof, Wolfgang (Hg.): Vom Fehlermachen und Lernen aus Fehlern: S. 11-41.

Reason, James (2008): Human Error. 19th printing. New York.

Wolff, Reinhart (1997): Kinderschutz auf dem Prüfstand. Überlegungen zur Notwendigkeit von Qualitätssicherung. In: Sternschnuppe 5. Kinderschutzzentrum Mainz.

Wolff, Reinhart (2009): Die Chancen dialogischer Qualitätsentwicklung und die Vision eines demokratischen Kinderschutzes. Vortrag auf der Auftaktveranstaltung des Projekts „Aus Fehlern lernen“ am 1.9.2009 in Berlin. Internet http://www.fruehehilfen.de/fileadmin/user_upload/fruehehilfen.de/pdf/Prof._Dr._Reinhart_Wolff_Vortrag_01.pdf

nehmerinnen, multiprofessionelle, interorganisationale Fall- und Fehleranalysen, Konzepte und Methoden der Qualitätssicherung und des Risikomanagements und die Entwicklung von Qualitätsstandards (insbesondere zur Zusammenarbeit und zur Qualitätssicherung und zum Fehlermanagement im Kinderschutz).

Nur wenn es gelingt, die Beteiligten vor Ort einzubeziehen und dialogisch vorzugehen, können wir eine fehleroffene Kultur erreichen, in der die Instrumente eines kritischen Fehlermanagements Früchte tragen (vgl. Wolff 2009). Auf diese Weise wird das Lernen aus Fehlern zur Chance, die wir nicht aufgeben sollten: $\mathrm{Zu}$ groß sind die Gewinne für Professionelle sowie für Nutzerinnen und Nutzer, wenn es (uns) gelingt (gerade im Kinderschutz), dialogisch aus Fehlern zu lernen.

\section{Anmerkungen}

(1) Zitiert nach: FAZ, 16.04.2010: »Bischof Mixa gesteht ein, geohrfeigt zu haben.«

(2) Allerdings hat die Arbeitsgruppe Kinderschutz an der Freien Universität Berlin, aus der dann das KinderschutzZentrum Berlin hervorging, bereits in den 1970er Jahren das Festhalten am weit verbreiteten "Züchtigungsrecht « als Gewalt gegen Kinder verurteilt. (Bast, H. u. a. 1975)

(3) Letzteres wird in der aktuellen Debatte um sogenannte "Risikofamilien « oft vergessen: Die Risikoeinschätzung suggeriert, es handele sich um natürlich selbst hervorgebrachte Risiken. Die gesellschaftliche Produktion der Risiken, in denen diese Familien leben, verschwindet im blinden Fleck der Wahrnehmung (vgl. Castel 2009. unter dem Stichwort der Naturalisierung von Risiken; siehe auch: Swift, K. J. und Callahan, M. 2009). 